\title{
Analytical Solution of General Bagley-Torvik Equation
}

\author{
William Labecca, Osvaldo Guimarães, and José Roberto C. Piqueira \\ Escola Politécnica da Universidade de São Paulo, Avenida Prof. Luciano Gualberto, Travessa 3, \\ No. 158, 05508-900 São Paulo, SP, Brazil \\ Correspondence should be addressed to José Roberto C. Piqueira; piqueira@lac.usp.br
}

Received 21 October 2015; Accepted 18 November 2015

Academic Editor: Ivan D. Rukhlenko

Copyright ( 2015 William Labecca et al. This is an open access article distributed under the Creative Commons Attribution License, which permits unrestricted use, distribution, and reproduction in any medium, provided the original work is properly cited.

\begin{abstract}
Bagley-Torvik equation appears in viscoelasticity problems where fractional derivatives seem to play an important role concerning empirical data. There are several works treating this equation by using numerical methods and analytic formulations. However, the analytical solutions presented in the literature consider particular cases of boundary and initial conditions, with inhomogeneous term often expressed in polynomial form. Here, by using Laplace transform methodology, the general inhomogeneous case is solved without restrictions in boundary and initial conditions. The generalized Mittag-Leffler functions with three parameters are used and the solutions presented are expressed in terms of Wiman's functions and their derivatives.
\end{abstract}

\section{Introduction}

Bagley-Torvik equations (BTE) firstly appeared in their seminal work [1] where they proposed to model viscoelastic behavior of geological strata, metals, and glasses by using fractional differential equations, showing that this approach is effective in describing structures containing elastic and viscoelastic components.

Initially, in [2], inhomogeneous BTE was studied with an analytical solution being proposed. Since then, there were several works to solve BTE, starting with numerical procedures for a reformulated BTE as a system of functional differential equations of order $1 / 2$ [3-5].

Following a numerical way for solving BTE, a generalization of Taylor's and Bessel's collocation method $[6,7]$ and the use of evolutionary computation [8] provided acceptable solutions from engineering point of view.

Approximation techniques were successfully applied to BTE mainly by using enhanced homotopic perturbation methods $[9,10]$, fractional iteration techniques [11], and cubic polynomial spline functions [12].

Analytical exact solutions for BTE were obtained in [13] for the particular initial condition $u(0)=u^{\prime}(0)=0$, considering the boundary condition given by $u(0)=u(1)=1$. Besides, by using a modified generalized Laguerre spectral method for fractional differential equations, BTE was solved in [14] for some specific conditions.

The aim of this work is to provide an analytical solution for the most generic case of inhomogeneous BTE in terms of the derivatives of Wiman's functions [2] considering that three-parameter generalized Mittag-Leffler function, introduced in [15], can be better explored.

The approach presented here consists of using the Laplace transform of Prabhakar's function, in order to solve the most general BTE, carrying out a subsequent transformation of the achieved solution in terms of the Mittag-Leffler functions.

In the next section, the BTE solution by using the referred to method is presented, followed by a section with the solution expressed by using Wiman's functions and their derivatives, motivated by the fact that Wiman's functions can be implemented in computational software like Mathematica ${ }^{\odot}$, easing the solution of BTE in practical applications.

\section{Laplace Transformation Solution of BTE: Mittag-Leffler Functions}

The most general inhomogeneous BTE [1] is given by

$$
A \mathscr{D}^{2} u(x)+B \mathscr{D}^{3 / 2} u(x)+C u(x)=\phi(x),
$$


which, by using operational notation, can be expressed as

$$
\left[A \mathscr{D}^{2}+B \mathscr{D}^{3 / 2}+C\right] u(x)=\phi(x) .
$$

Here the domain of $\phi(x)$ is considered to be the time; consequently it is assumed that $t \in[0, \infty)$. The Riemann-Liouville fractional integral is adopted and, therefore, Caputo's fractional derivative ${ }^{c} D_{0}^{v}$ can be simply denoted by $\mathscr{D}[16,17]$.

The method to be used to solve BTE is the traditional Laplace transform that, due to the fractional nature of the exponents, results from Prabhakar functions, that is, threeparameter Mittag-Leffler generalized functions $[2,15]$.

Considering Caputo's fractional derivative [18], the Laplace transform of the fractional exponent term is

$$
\mathscr{L}\left\{\mathscr{D}^{3 / 2} u(x)\right\}=s^{3 / 2} U(s)-s^{1 / 2} u(0)-s^{-1 / 2} u^{\prime}(0),
$$

implying that the Laplace transform of the BTE is written as

$$
\begin{aligned}
& U(s)\left(A s^{2}+B s^{3 / 2}+C\right) \\
& \quad=\left(A s+B s^{1 / 2}\right) u(0)+\left(B s^{-1 / 2}+A\right) u^{\prime}(0)+\Phi(s) .
\end{aligned}
$$

Defining $B / A=\lambda$ and $C / A=\mu$ and considering the binomial development,

$$
\begin{aligned}
\left(1+\frac{\lambda s^{3 / 2}}{s^{2}+\mu}\right)^{-1} & =\left(1+\frac{B s^{3 / 2}}{A s^{2}+C}\right)^{-1} \\
& =\sum_{k=0}^{\infty}(-1)^{k} \frac{\lambda^{k} s^{3 k / 2}}{\left(s^{2}+\mu\right)^{k}}
\end{aligned}
$$

the Laplace transform of BTE's solution is

$$
\begin{aligned}
& U(s)=\sum_{k=0}^{\infty}(-1)^{k} \lambda^{k} \frac{s^{3 k / 2}}{\left(s^{2}+\mu\right)^{k+1}}\left[\left(s+\lambda s^{1 / 2}\right) u(0)\right. \\
& \left.+\left(\lambda s^{-1 / 2}+1\right) u^{\prime}(0)+\frac{1}{A} \Phi(s)\right] .
\end{aligned}
$$

In order to invert the Laplace transform to obtain the general BTE's solution, $U(s)$ is rewritten expanding the sums:

$$
\begin{aligned}
U(s)= & u(0) \sum_{k=0}^{\infty}(-1)^{k} \lambda^{k} \frac{s^{3 k / 2+1}}{\left(s^{2}+\mu\right)^{k+1}} \\
& +u(0) \sum_{k=0}^{\infty}(-1)^{k} \lambda^{k+1} \frac{s^{3 k / 2+1 / 2}}{\left(s^{2}+\mu\right)^{k+1}} \\
& +u^{\prime}(0) \sum_{k=0}^{\infty}(-1)^{k} \lambda^{k+1} \frac{s^{3 k / 2-1 / 2}}{\left(s^{2}+\mu\right)^{k+1}} \\
& +u^{\prime}(0) \sum_{k=0}^{\infty}(-1)^{k} \lambda^{k} \frac{s^{3 k / 2}}{\left(s^{2}+\mu\right)^{k+1}} \\
& +\frac{1}{A} \Phi(s) \sum_{k=0}^{\infty}(-1)^{k} \lambda^{k} \frac{s^{3 k / 2}}{\left(s^{2}+\mu\right)^{k+1}}
\end{aligned}
$$

As shown in $[2,15]$, it is possible to define three-parameter Mittag-Leffler general functions as

$$
\begin{aligned}
E_{\alpha, \beta}^{\gamma}(t):= & \sum_{k=0}^{\infty} \frac{(\gamma)_{k}\left(t^{k}\right)}{\Gamma(\alpha k+\beta) k !}, \\
& (\alpha, \beta, \gamma \in \mathscr{C}, \mathfrak{R}(\alpha), \mathfrak{R}(\beta), \mathfrak{R}(\gamma)>0),
\end{aligned}
$$

with $(\mu)_{k}=\Gamma(\mu+k) / \Gamma(\mu)$ being the Pochhammer symbol. When $\gamma=1$, these functions are called Wiman's functions.

Here, the main fact to be used is related to the inverse of the Laplace transform of the main terms that appear in (7), given by

$$
\mathscr{L}^{-1}\left\{\frac{s^{\alpha \gamma-\beta}}{\left(s^{\alpha}+\lambda\right)^{\gamma}}\right\}=x^{\beta-1} E_{\alpha, \beta}^{\gamma}\left(-\lambda x^{\alpha}\right) .
$$

Consequently, the first four terms of (7) can be inverted and written as

$$
\begin{aligned}
& \mathscr{L}^{-1}\left\{\frac{s^{3 k / 2+1}}{\left(s^{2}+\mu\right)^{k+1}}\right\}=x^{(1 / 2) k} E_{2,(1 / 2)(k+2)}^{k+1}\left(-\mu x^{2}\right), \\
& \mathscr{L}^{-1}\left\{\frac{s^{3 k / 2+1 / 2}}{\left(s^{2}+\mu\right)^{k+1}}\right\}=x^{(1 / 2)(k+1)} E_{2,(1 / 2)(k+3)}^{k+1}\left(-\mu x^{2}\right), \\
& \mathscr{L}^{-1}\left\{\frac{s^{3 k / 2-1 / 2}}{\left(s^{2}+\mu\right)^{k+1}}\right\}=x^{(1 / 2)(k+3)} E_{2,(1 / 2)(k+5)}^{k+1}\left(-\mu x^{2}\right), \\
& \mathscr{L}^{-1}\left\{\frac{s^{3 k / 2}}{\left(s^{2}+\mu\right)^{k+1}}\right\}=x^{(1 / 2)(k+2)} E_{2,(1 / 2)(k+4)}^{k+1}\left(-\mu x^{2}\right) .
\end{aligned}
$$
tion:

The inhomogeneous term is calculated by using convolu-

$$
\begin{aligned}
\mathscr{L}^{-1} & \left\{\frac{\Phi(s) s^{3 k / 2}}{\left(s^{2}+\mu\right)^{k+1}}\right\} \\
= & \phi(x) *\left[x^{(1 / 2)(k+2)} E_{2,(1 / 2)(k+4)}^{k+1}\left(-\mu x^{2}\right)\right],
\end{aligned}
$$

and, consequently, the general solution of BTE is given by

$$
\begin{aligned}
& u(x)=u(0) \sum_{k=0}^{\infty}(-\lambda)^{k} x^{k / 2} E_{2,(1 / 2)(k+2)}^{k+1}\left(-\mu x^{2}\right)+u(0) \\
& \cdot \sum_{k=0}^{\infty}(-\lambda)^{k} x^{(1 / 2)(k+1)} E_{2,(1 / 2)(k+3)}^{k+1}\left(-\mu x^{2}\right)+u^{\prime}(0) \\
& \cdot \sum_{k=0}^{\infty}(-\lambda)^{k} x^{(1 / 2)(k+2)} E_{2,(1 / 2)(k+4)}^{k+1}\left(-\mu x^{2}\right)+u^{\prime}(0) \\
& \cdot \sum_{k=0}^{\infty}(-\lambda)^{k} x^{(1 / 2)(k+3)} E_{2,(1 / 2)(k+5)}^{k+1}\left(-\mu x^{2}\right)+\frac{1}{A} \\
& \quad \cdot \sum_{k=0}^{\infty}(-\lambda)^{k} \\
& \quad \int_{0}^{x} \phi(x-\xi) \xi^{(1 / 2)(k+2)} E_{2,(1 / 2)(k+4)}^{k+1}\left(-\mu \xi^{2}\right) d \xi .
\end{aligned}
$$


In order to simplify the BTE general solution expressed by (12), a factor $\rho(s)$ can be defined:

$$
\rho(s):=\frac{1}{2}\left\{1-(-1)^{[4 / s]}\right\}
$$

with $[k] \equiv \operatorname{int}(k)$. Therefore the BTE solution is expressed as

$$
\begin{aligned}
& u(x)=\sum_{k=0}^{\infty}\left(-\frac{B}{A}\right)^{k}\left\{\left.\sum_{s=1}^{4} D^{\rho(s)} u(x)\right|_{x=0}\right. \\
& \cdot x^{(1 / 2)(k+s-1)} E_{2,(1 / 2)(k+s+1)}^{k+1}\left(-\frac{C x^{2}}{A}\right)+\frac{1}{A} \\
& \cdot \int_{0}^{x} \phi(x-\xi) \\
& \left.\cdot \xi^{(1 / 2)(k+2)} E_{2,(1 / 2)(k+4)}^{k+1}\left(-\frac{C}{A} \xi^{2}\right) d \xi\right\} .
\end{aligned}
$$

Operator $D^{\rho(s)}$ represents the integer order classical derivative, that is, $D^{\rho} \equiv(d / d x)^{\rho}$, with the property $D^{0} u(x) \equiv$ $u(x)$.

Expression (14) is a general analytical solution of BTE in terms of three-parameter Mittag-Leffler functions, $E_{\alpha, \beta}^{\gamma}(x)$. However, the solution established in [2] for the special case with $u(0)=u^{\prime}(0)=0$ is given by using Wiman's functions derivatives, that is, two-parameter Mittag-Leffler functions.

As Wiman's functions and derivatives are part of several software packages, as Mathematica ${ }^{\odot}$, for instance, and threeparameter Mittag-Leffler are not, the results will be modified to be compatible with this fact, easing the practical approach of the problem.

\section{BTE Solution via Wiman's Functions and Derivatives}

Here, the solution of BTE expressed by (14) is modified, in order to be written in terms of Wiman's functions and their derivatives. The explicit forms of these functions are given by

$$
\begin{array}{r}
E_{\lambda, \mu}^{(k)}(y) \equiv \frac{d^{k}}{d y^{k}} E_{\lambda, \mu}(y)=\sum_{j=0}^{\infty} \frac{(j+k) ! y^{j}}{j ! \Gamma(\lambda j+\lambda k+\mu)}, \\
(k=0,1,2, \ldots) .
\end{array}
$$

For the sake of clearness, the work will be divided into two parts: homogeneous solution terms and inhomogeneous solution term.

3.1. Homogeneous Solution Terms. Taking the homogeneous part of (14), denoted by $h(x)$,

$$
\begin{aligned}
h(x) & =\sum_{k=0}^{\infty}\left(-\frac{B}{A}\right)^{k} \\
& \left.\cdot \sum_{s=1}^{4} D^{\rho(s)} u(x)\right|_{x=0} x^{(1 / 2)(k+s-1)} E_{2,(1 / 2)(k+s+1)}^{k+1}
\end{aligned}
$$

$$
\begin{aligned}
& \cdot\left(-\frac{C x^{2}}{A}\right)=\sum_{k=0}^{\infty}\left(-\frac{B}{A}\right)^{k} \\
& \left.\cdot \sum_{s=1}^{4} D^{\rho(s)} u(x)\right|_{x=0} x^{(1 / 2)(k+s-1)} \\
& \cdot \sum_{r=0}^{\infty} \frac{(k+r) !(-1)^{r}(C / A)^{r} x^{2 r}}{k ! \Gamma(2 r+k / 2+s / 2+1 / 2)} .
\end{aligned}
$$

If the $r$ and $k$ sums are uniformly convergent,

$$
\begin{gathered}
h(x)=\left.\sum_{r=0}^{\infty} \frac{(-1)^{r}}{r !}\left(\frac{C}{A}\right)^{r} \sum_{s=1}^{4} D^{\rho(s)} u(x)\right|_{x=0} x^{2 r+(1 / 2)(s-1)}, \\
\sum_{k=0}^{\infty} \frac{(k+r) !}{k ! \Gamma[r / 2+k / 2+(1 / 2)(3 r+s+1)]}\left(-\frac{B \sqrt{x}}{A}\right)^{k} .
\end{gathered}
$$

Observing that the last sum can be written as the $r$ th derivative of Wiman's function, the homogeneous part of the solution is

$$
\begin{gathered}
h(x)=\left.\sum_{r=0}^{\infty} \frac{(-1)^{r}}{r !}\left(\frac{C}{A}\right)^{r} \sum_{s=1}^{4} D^{\rho(s)} u(x)\right|_{x=0} \\
\cdot x^{2 r+(1 / 2)(s-1)} E_{1 / 2,(1 / 2)(3 r+s+1)}^{(r)}\left(-\frac{B \sqrt{x}}{A}\right) .
\end{gathered}
$$

3.2. Inhomogeneous Solution Term. Calling the inhomogeneous term of the BTE solution $\psi(x)$, its expression is

$$
\begin{aligned}
& \psi(x)=\sum_{k=0}^{\infty}\left(-\frac{B}{A}\right)^{k} \frac{1}{A} \\
& \cdot \int_{0}^{x} \phi(x-\xi) \xi^{(1 / 2)(k+2)} E_{2,(1 / 2)(k+4)}^{k+1}\left(-\frac{C}{A} \xi^{2}\right) d \xi \\
& =\sum_{k=0}^{\infty}\left(-\frac{B}{A}\right)^{k} \frac{1}{A} \int_{0}^{x} \phi(x-\xi) \xi^{k / 2+1} \\
& \cdot \sum_{r=0}^{\infty} \frac{(-1)^{r}(k+1)_{r}}{r ! \Gamma(2 r+k / 2+2)}\left(\frac{C}{A} \xi^{2}\right)^{r} d \xi .
\end{aligned}
$$

Considering the convergence of the integral and uniform convergence of the sums, their order can be changed and it can be written as

$$
\begin{aligned}
& \psi(x)=\int_{0}^{x} \phi(x-\xi) \sum_{r=0}^{\infty} \frac{(-1)^{r}}{A r !}\left(\frac{C}{A}\right)^{r} \xi^{2 r+1} \\
& \quad \cdot \sum_{k=0}^{\infty} \frac{(-1)^{k} \Gamma(k+r+1) \xi^{k / 2}}{\Gamma(k+1) \Gamma(2 r+k / 2+2)}\left(\frac{B}{A}\right)^{k} d \xi \\
& =\int_{0}^{x} \phi(x-\xi) \frac{1}{A} \sum_{r=0}^{\infty} \frac{(-1)^{r}}{r !}\left(\frac{C}{A}\right)^{r} \xi^{2 r+1} \\
& \quad \cdot \sum_{k=0}^{\infty} \frac{(k+r) !}{k !} \frac{(-B \sqrt{\xi} / A)^{k}}{\Gamma[(1 / 2) r+(1 / 2) k+((3 / 2) r+2)]} d \xi
\end{aligned}
$$


It can be noticed that the last sum of (20) can be written as the $r$-order derivative of Wiman's function and, consequently,

$$
\begin{aligned}
& \psi(x)=\int_{0}^{x} \phi(x-\xi) \frac{1}{A} \\
& \cdot \sum_{r=0}^{\infty} \frac{(-1)^{r}}{r !}\left(\frac{C}{A}\right)^{r} \xi^{2 r+1} E_{1 / 2,2+(3 / 2) r}^{(r)}\left(-\frac{B}{A} \sqrt{\xi}\right) d \xi
\end{aligned}
$$

Then, defining

$$
G(\xi)=\frac{1}{A} \sum_{r=0}^{\infty} \frac{(-1)^{r}}{r !}\left(\frac{C}{A}\right)^{r} \xi^{2 r+1} E_{1 / 2,2+(3 / 2) r}^{(r)}\left(-\frac{B}{A} \sqrt{\xi}\right),
$$

it follows that

$$
\psi(x)=\int_{0}^{x} \phi(x-\xi) G(\xi) d \xi
$$

according to the BTE analytical solution obtained in [2] for $u(0)=u^{\prime}(0)=0$.

3.3. Complete BTE Solution. Considering that $u(x)=h(x)+$ $\psi(x)$, the general analytical solution of BTE, in terms of Wiman's function, is

$$
\begin{aligned}
& u(x)=\sum_{r=0}^{\infty} \frac{(-1)^{r}}{r !}\left(\frac{C}{A}\right)^{r}\left\{\left.\sum_{s=1}^{4} D^{\rho(s)} u(x)\right|_{x=0}\right. \\
& \cdot x^{2 r+(1 / 2)(s-1)} E_{1 / 2,(1 / 2)(3 r+s+1)}^{(r)}\left(-\frac{B \sqrt{x}}{A}\right)+\frac{1}{A} \\
& \left.\cdot \int_{0}^{x} \phi(x-\xi) \xi^{2 r+1} E_{1 / 2,2+(3 / 2) r}^{(r)}\left(-\frac{B}{A} \sqrt{\xi}\right) d \xi\right\},
\end{aligned}
$$

with $\rho(s):=(1 / 2)\left\{1-(-1)^{[4 / s]}\right\}$ and $E_{\alpha, \beta}^{(r)}(z)=$ $\left(d^{r} / d z^{r}\right) E_{\alpha, \beta}(z)$.

\section{Conclusion}

A general analytical solution of BTE defined by Caputo's fractional derivatives was obtained in terms of Wiman's functions and their derivatives. The reasoning was conducted by using Laplace transform and Mittag-Leffler functions.

The final expression permits the calculations in practical cases as it is built considering Wiman's functions that are part of the most usual numerical packages.

\section{Conflict of Interests}

The authors declare that there is no conflict of interests regarding the publication of this paper.

\section{References}

[1] P. J. Torvik and R. L. Bagley, "On the appearance of the fractional derivative in the behavior of real materials," Journal of Applied Mechanics, vol. 51, no. 2, pp. 294-298, 1984.
[2] I. Podlubny, Fractional Differential Equations, Academic Press, San Diego, Calif, USA, 1999.

[3] A. H. Bhrawy, T. M. Taha, and J. A. T. Machado, "A review of operational matrices and spectral techniques for fractional calculus," Nonlinear Dynamics, vol. 81, no. 3, pp. 1023-1052, 2015.

[4] K. Diethelm and N. J. Ford, "Numerical solution of the BagleyTorvik equation," BIT. Numerical Mathematics, vol. 42, no. 3, pp. 490-507, 2002.

[5] Z. H. Wang and X. Wang, "General solution of the BagleyTorvik equation with fractional-order derivative," Communications in Nonlinear Science and Numerical Simulation, vol. 15, no. 5, pp. 1279-1285, 2010.

[6] Y. Çenesiz, Y. Keskin, and A. Kurnaz, "The solution of the Bagley-Torvik equation with the generalized TAYlor collocation method," IEEE Engineering and Applied Mathematics, vol. 347, no. 2, pp. 452-466, 2010.

[7] Ş. Yüzbaşı, "Numerical solution of the Bagley-Torvik equation by the Bessel collocation method," Mathematical Methods in the Applied Sciences, vol. 36, no. 3, pp. 300-312, 2013.

[8] M. A. Raja, J. A. Khan, and I. M. Qureshi, "Solution of fractional order system of Bagley-Torvik equation using evolutionary computational intelligence," Mathematical Problems in Engineering, vol. 2011, Article ID 675075, 18 pages, 2011.

[9] F. Abidi and K. Omrani, "The homotopy analysis method for solving the Fornberg-Whitham equation and comparison with Adomian's decomposition method," Computers \& Mathematics with Applications, vol. 59, no. 8, pp. 2743-2750, 2010.

[10] M. Zolfaghari, R. Ghaderi, A. SheikholEslami et al., "Application of the enhanced homotopy perturbation method to solve the fractional-order Bagley-Torvik differential equation," Physica Scripta, vol. 2009, article T136, Article ID 014032, 2009.

[11] T. Mekkaoui and Z. Hammouch, "Approximate analytical solutions to the Bagley-Torvik equation by the fractional iteration method," Annals of the University of Craiova-Mathematics and Computer Science Series, vol. 39, no. 2, pp. 251-256, 2012.

[12] W. K. Zahra and S. M. Elkholy, "Cubic spline solution of fractional Bagley-Torvik equation," Electronic Journal of Mathematical Analysis and Applications, vol. 1, no. 2, pp. 230-241, 2013.

[13] D. Baleanu, A. H. Bhrawy, and T. M. Taha, "A modified generalized Laguerre spectral method for fractional differential equations on the half line," Abstract and Applied Analysis, vol. 2013, Article ID 413529, 12 pages, 2013.

[14] S. Staněk, "Two-point boundary value problems for the generalized Bagley-Torvik fractional differential equation," Central European Journal of Mathematics, vol. 11, no. 3, pp. 574-593, 2013.

[15] T. R. Prabhakar, "A singular integral equation with a generalized Mittag-Leffler function in the kernel," Yokohama Mathematical Journal, vol. 19, pp. 7-15, 1971.

[16] K. M. Kolwankar and A. D. Gangal, "Fractional differentiability of nowhere differentiable functions and dimensions," Chaos, vol. 6, no. 4, pp. 505-513, 1996.

[17] T. Kisela, Fractional differential equations and their applications [M.S. thesis], University of l'Aquila \& Brno University of Technology, Brno, Czech Republic, 2008.

[18] A. M. O. Anwar, F. Jarad, D. Baleanu, and F. Ayaz, "Fractional Caputo heat equation within the double Laplace transform," Romanian Journal of Physics, vol. 58, no. 1-2, pp. 15-22, 2013. 


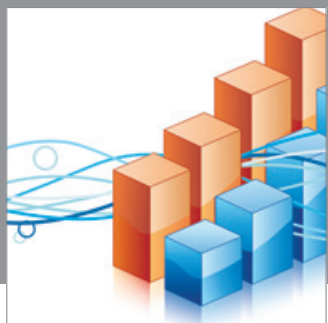

Advances in

Operations Research

mansans

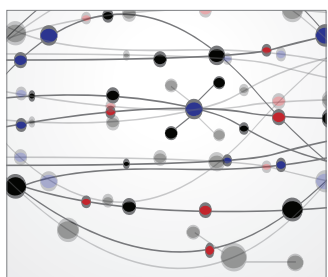

The Scientific World Journal
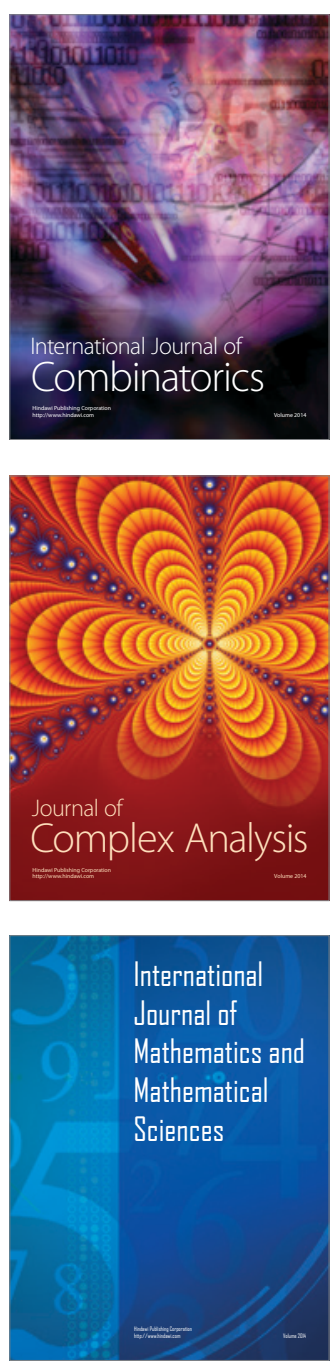
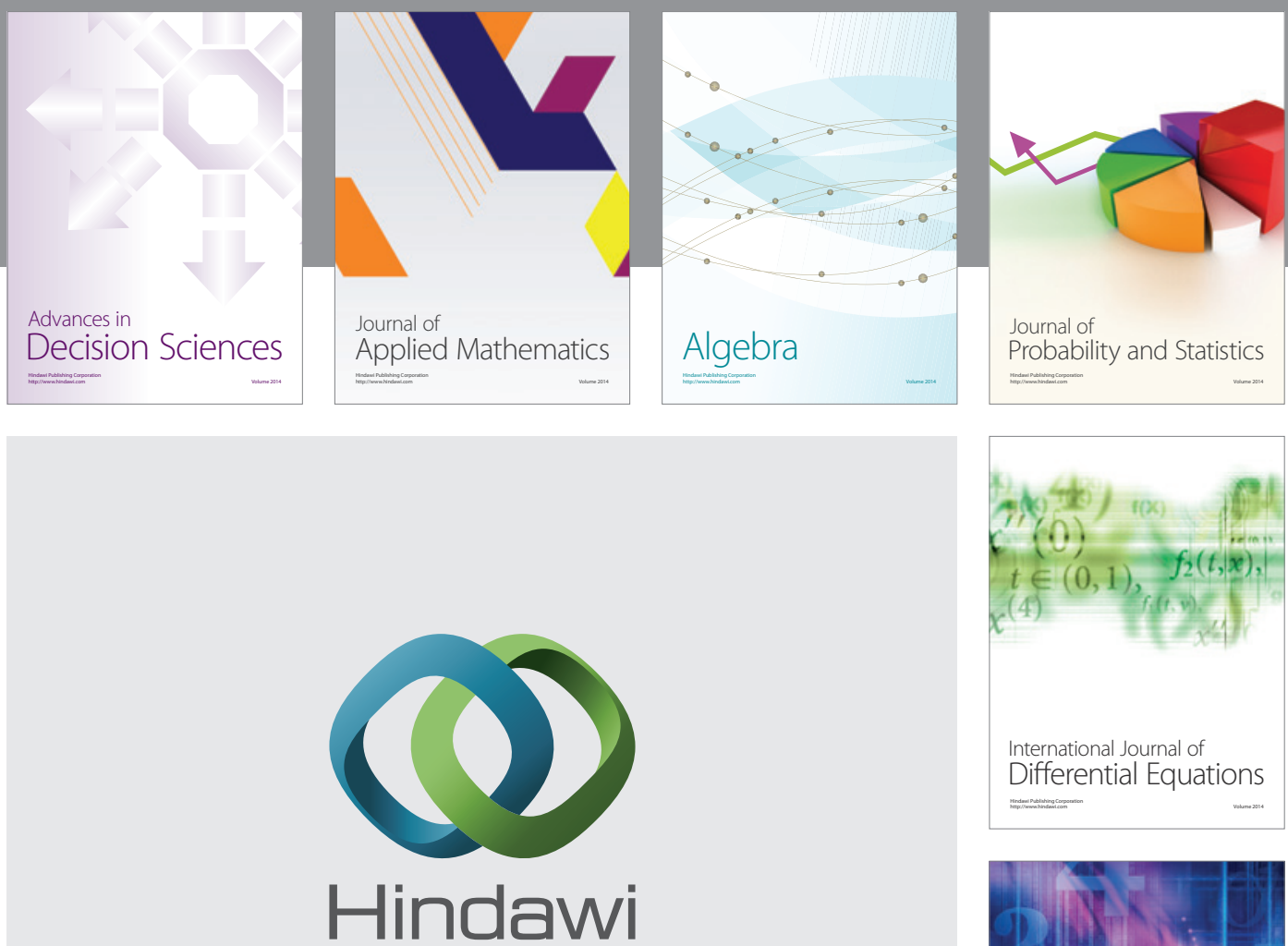

Submit your manuscripts at http://www.hindawi.com
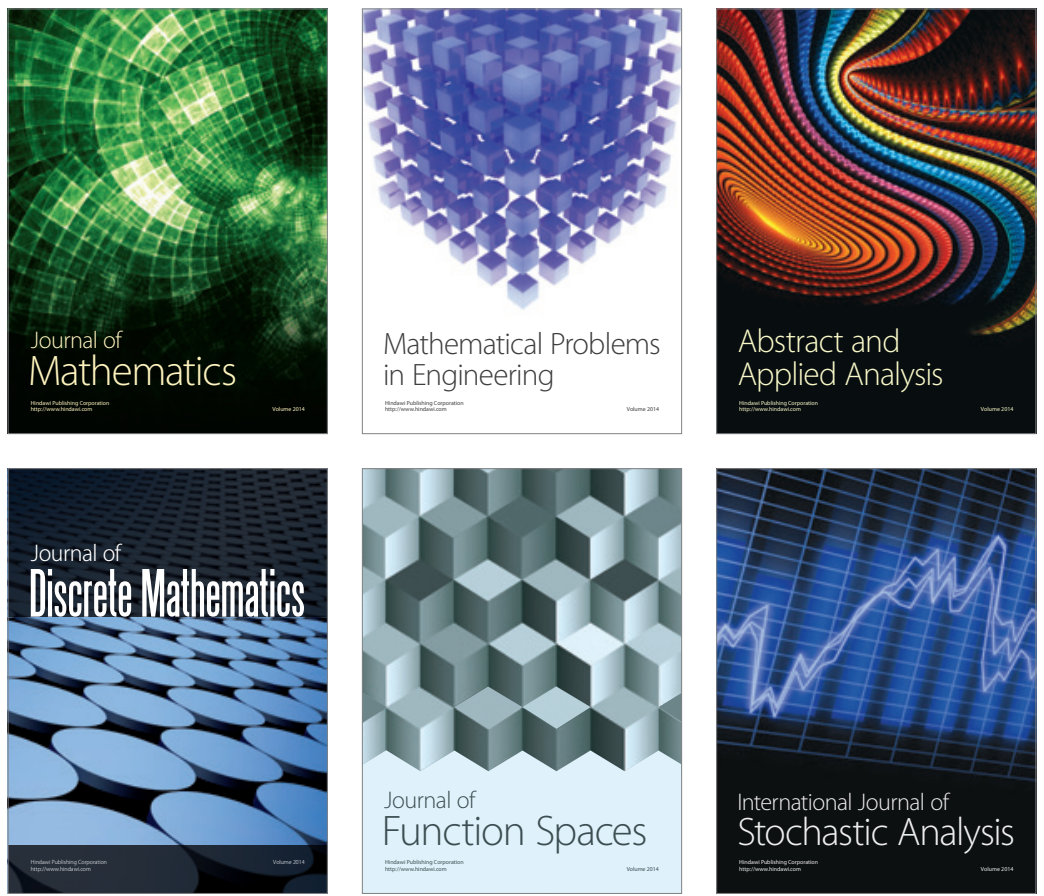

Journal of

Function Spaces

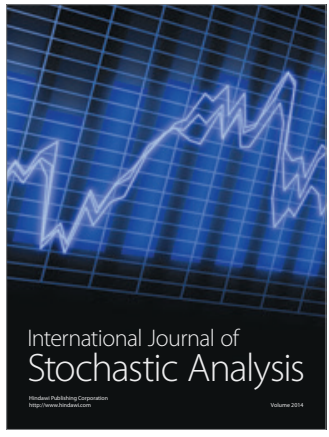

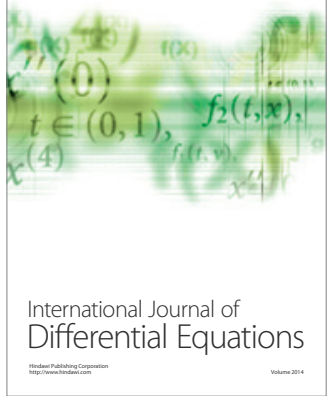
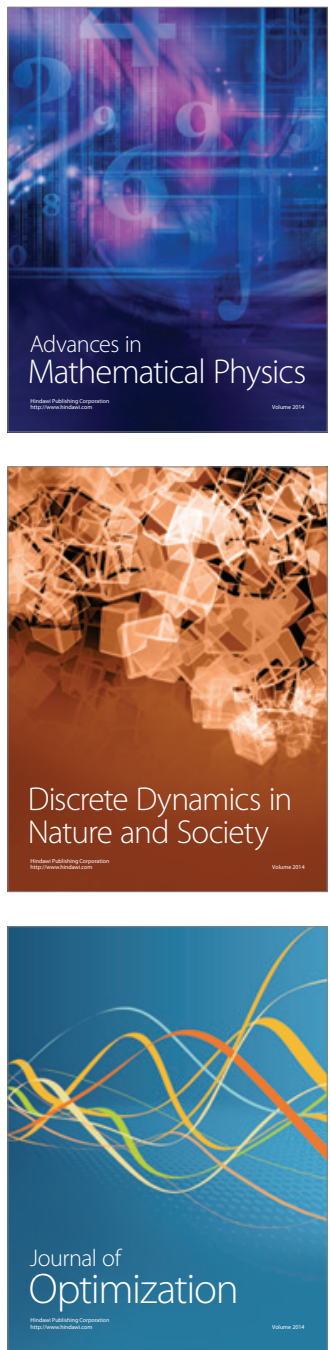